\title{
Effect of [111] texture on the perpendicular magnetic anisotropy of $\mathrm{Co} / \mathrm{Ni}$ multilayers
}

\author{
V. M. Naik \\ Department of Natural Sciences, University of Michigan-Dearborn, Dearborn, Michigan 48128 \\ S. Hameed, R. Naik, L. Pust, L. E. Wenger, and G. L. Dunifer \\ Department of Physics \& Astronomy, Wayne State University, Detroit, Michigan 48202 \\ G. W. Auner \\ Department of Electrical \& Computer Engineering, Wayne State University, Detroit, Michigan 48202
}

(Received 27 April 1998; accepted for publication 19 June 1998)

$[111]_{\text {fcc }}$ oriented $[\mathrm{Co}(2 \AA) / \mathrm{Ni}(7 \AA)]_{20}$ multilayers were prepared by molecular beam epitaxy at room temperature on epitaxial $\mathrm{Au} / \mathrm{Ag}$ buffer layers grown on chemically etched $\mathrm{Si}(111)$ surfaces. $\mathrm{NH}_{4} \mathrm{~F}$ etching of $\mathrm{Si}(111)$ leads to a smaller spread in the $\langle 111\rangle$ orientation of the $\mathrm{Au} / \mathrm{Ag}$ buffer layers and the $\mathrm{Co} / \mathrm{Ni}$ multilayers as compared to a similar sample prepared on HF-etched $\mathrm{Si}(111)$. This results in a stronger perpendicular magnetic anisotropy as determined from the magnetic hysteresis loops. Cross-sectional transmission electron microscopy studies show that the magnetic multilayer film is not flat but has significant waviness at both top and bottom surfaces. The observed waviness originates in part from the waviness already present on the surface of the Au buffer layer and appears to be further enhanced by the difference in the surface free energies of $\mathrm{Au}$ and the magnetic elements. A flatter $\mathrm{Au} / \mathrm{Ag}$ buffer layer is essential to further improve the [111] texture of the $[\mathrm{Co} / \mathrm{Ni}]$ multilayers. (C) 1998 American Institute of Physics. [S0021-8979(98)06718-8]

\section{INTRODUCTION}

From ab initio calculations (local-spin density approximation) of the magnetocrystalline anisotropy energy, Daalderop et al. ${ }^{1}$ recently predicted and demonstrated a perpendicular orientation for the magnetization in $[111]_{\mathrm{fcc}} \mathrm{Co}_{1} / \mathrm{Ni}_{2}$ multilayer (ML) structures. Their $[\mathrm{Co}(2 \AA) / \mathrm{Ni}(4 \AA)]_{20} \mathrm{ML}$ structure showed a perpendicular magnetic anisotropy (PMA) with a saturation magnetization of $1 \mathrm{~T}$ which is much larger than that of any known PMA multilayer consisting of a magnetic and a nonmagnetic metal, e.g., $\mathrm{Co} / \mathrm{Pt}, \mathrm{Co} / \mathrm{Pd}$, $\mathrm{Co} / \mathrm{Ir}$, etc. ${ }^{2}$ In fact, the PMA exists for a $\mathrm{Co} / \mathrm{Ni} \mathrm{ML}$ with $t_{\mathrm{Co}}=2 \AA$ and $t_{\mathrm{Ni}}$ between 2 and $\sim 12 \AA$. Furthermore their work ${ }^{1}$ clearly demonstrated that a large interface anisotropy could occur even when both elements were magnetic. The magneto-optical properties of $\mathrm{Co}_{1} / \mathrm{Ni}_{2} \mathrm{ML}$ samples prepared by high vacuum vapor deposition on a glass substrate with metallic underlayers $(\mathrm{Au}, \mathrm{Cu})$ have been reported by den Broeder et $\mathrm{al}^{3}{ }^{3}$ For certain deposition conditions, the polar magneto-optic hysteresis loop of a $120 \AA \mathrm{Co}_{1} / \mathrm{Ni}_{2}$ ML exhibited $100 \%$ remanence with a coercivity as high as 140 $\mathrm{kA} / \mathrm{m}$. On the other hand a codeposited Co-Ni alloy film with similar thickness, composition, and growth conditions showed a strong in-plane anisotropy. This observed difference in magnetic anisotropy is a clear indication that the PMA results from [111] oriented $\mathrm{Co} / \mathrm{Ni}$ interfaces.

In the present work, we have carried out a systematic study to determine the effect of the [111] texture on the PMA of a $\mathrm{Co} / \mathrm{Ni}$ ML. $[111]_{\mathrm{fcc}}$ oriented $[\mathrm{Co}(2 \AA) / \mathrm{Ni}(7 \AA)]_{20}$ multilayer films have been fabricated by molecular beam epitaxy (MBE) at room temperature on epitaxial $\mathrm{Au}(100 \AA) /$ $\operatorname{Ag}(100 \AA)$ buffer layers grown on chemically etched $\mathrm{Si}(111)$ surfaces. Two different methods of chemical etching were used prior to loading the $\mathrm{Si}(111)$ substrates into the load lock of the MBE deposition system: (i) etching with a $10 \% \mathrm{HF}$ solution and (ii) etching with a $40 \% \mathrm{NH}_{4} \mathrm{~F}$ solution. Previous studies $^{4,5}$ have shown that these processes passivate the $\mathrm{Si}(111)$ surface against oxidation by hydrogen termination of the dangling Si bonds. In addition, scanning tunneling microscopy studies have shown that etching with a dilute HF solution produces surfaces with microscopic roughness $(3 \AA)$ whereas etching with $\mathrm{NH}_{4} \mathrm{~F}$ results in atomically smooth surfaces over areas on the order of $1000 \AA \times 1000 \AA .{ }^{4}$ We have used these two different methods of chemical etching of the $\mathrm{Si}$ (111) surface in order to obtain epitaxial $\mathrm{Au} / \mathrm{Ag}$ buffer layers with different [111] texture and correspondingly different texture for the Co/Ni ML. Structural characterizations were performed using in situ reflection high energy electron diffraction (RHEED) during the growth process and x-ray diffraction (XRD)/rocking curves to quantitatively determine the degree of [111] texture, while the interfacial/surface features and epitaxial orientation relationships between $\mathrm{Ag} / \mathrm{Si}$, $\mathrm{Au} / \mathrm{Ag},(\mathrm{Co} / \mathrm{Ni}) \mathrm{ML} / \mathrm{Au}$ were investigated by cross-sectional transmission electron microscopy (TEM). Also the surface morphology of the films was examined by atomic force microscopy (AFM) to explore any possible correlation to the PMA properties of the films.

\section{EXPERIMENT}

In an earlier study, the growth of epitaxial $\operatorname{Ag}(111)$ films on HF-etched $\mathrm{Si}(111)$ surfaces at room temperature as well as the details of substrate preparation, growth conditions, epitaxial orientation relations, etc., were described. ${ }^{6}$ Briefly, these films were grown in an ultrahigh vacuum environment using an MBE deposition system with a base pressure of 
better than $2 \times 10^{-10}$ Torr. $\mathrm{Si}(111)$ substrates were etched either in a $10 \% \mathrm{HF}$ solution for about $30 \mathrm{~s}$ or in a $\mathrm{NH}_{4} \mathrm{~F}$ solution for about $5 \mathrm{~min}$ and then rinsed in deionized water. In both cases the substrates were pulled slowly from the solution so as to have visibly clean surfaces without any wet spots. The evaporation rate was approximately $0.05 \mathrm{~nm} / \mathrm{s}$ based on a quartz crystal thickness monitor which was calibrated using a diamond stylus profilometer. The measured thickness has an error of $\pm 10 \%$. RHEED patterns were continuously monitored during the deposition to gauge the quality and surface structure of the films. Fabrication of the samples involved the growth of an initial $100-\AA$-thick, epitaxial $\operatorname{Ag}(111)$ layer on a hydrogen-terminated $\mathrm{Si}(111)$ surface (sample No. 1 with HF etching and sample No. 2 with $\mathrm{NH}_{4} \mathrm{~F}$ etching) at room temperature followed by a $100-\AA$ thick, $\mathrm{Au}(111)$ layer, and then the epitaxial $[\mathrm{Co}(2 \AA) / \mathrm{Ni}(7 \AA)]_{20}$ ML. A final 20-Å-thick, Au cap layer was deposited to prevent oxidation. A third sample No. 3 was prepared with a thicker buffer layer of $\mathrm{Au}(500 \AA)$ / $\mathrm{Ag}(500 \AA)$ on $\mathrm{NH}_{4} \mathrm{~F}$-etched $\mathrm{Si}(111)$ in order to determine the effect of a thicker buffer layer on the [111] texture of the magnetic ML.

Standard x-ray diffraction $(\theta-2 \theta)$ scans and rocking curves were performed with a Rigaku powder diffractometer using $\mathrm{Cu} K \alpha$ radiation. The surface morphology of the films was examined by a Digital Instruments Nanoscope III Multimode atomic force microscope operating in the contact mode. TEM cross-sectional samples were fabricated by bonding two films face to face, mechanical thinning, and then ion milling. Atomic resolution TEM was performed on a JEOL 4000EX transmission electron microscope operating at $400 \mathrm{keV}$. Magnetization measurements were done at room temperature using a Quantum Design superconducting quantum interference device (SQUID) magnetometer with magnetic fields applied both parallel and perpendicular to the film plane in order to elucidate the PMA properties.

\section{RESULTS AND DISCUSSION}

Figure 1 shows the RHEED patterns observed along the $\langle 110\rangle$ azimuth of $\mathrm{Si}$ at the initial stages of the $\mathrm{Ag}$ growth on the HF-etched $\mathrm{Si}\left(111\right.$ ) (sample No. 1) and $\mathrm{NH}_{4} \mathrm{~F}$-etched $\mathrm{Si}(111)$ (sample No. 2) substrates. During the initial growth of $\mathrm{Ag}$ the diffraction spots are sharper for the $\mathrm{NH}_{4} \mathrm{~F}$-etched $\mathrm{Si}(111)$ substrate [see Fig. 1(e)] as compared to the HFetched $\mathrm{Si}(111)$ substrate [see Fig. 1(b)] indicating a better orientation, although at later stages the RHEED patterns look very similar [Figs. 1(c) and 1(f)]. The RHEED patterns indicate that the growth of $\mathrm{Ag}$ on $\mathrm{Si}(111)$ is epitaxial with $\operatorname{Ag}(111) \| \operatorname{Si}(111)$ and $[110]_{\mathrm{Ag}} \|[110]_{\mathrm{Si}}$. In addition, the closely spaced spots observed on the vertical streaks indicate the presence of $180^{\circ}$ twins of epitaxial Ag crystallites about the $\mathrm{Ag}(111)$ planes. ${ }^{6} \mathrm{X}$-ray diffraction (XRD) measurements further confirm the [111] texture of the films as only (111) peaks of the $\mathrm{Au} / \mathrm{Ag}$ buffer layers $\left(2 \theta \sim 38.2^{\circ}\right)$ and the $\mathrm{Co} / \mathrm{Ni}$ ML $\left(2 \theta \sim 44.3^{\circ}\right)$ are present. The rocking curve data presented as full width at half maximum (FWHM) of the (111) diffraction peaks for both the $\mathrm{Au} / \mathrm{Ag}$ buffer layers and $\mathrm{Co} / \mathrm{Ni}$ $\mathrm{ML}$ are shown in Table I. The large values of the FWHM are
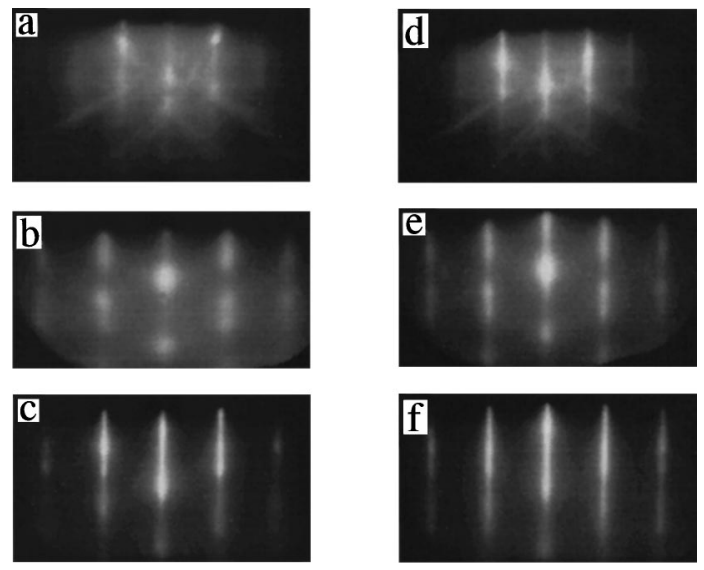

FIG. 1. RHEED patterns during Ag deposition at room temperature: (a)-(c) correspond to the HF-etched $\mathrm{Si}(111)$ substrate and (d)-(f) correspond to the $\mathrm{NH}_{4}$ F-etched $\mathrm{Si}(111)$ substrate. (a) and (d) show patterns obtained from Si(111) substrate viewed along $\langle 110\rangle$ azimuth; (b) and (e) after a $3 \AA \mathrm{Ag}$ deposition; (c) and (f) after $100 \AA$ Ag deposition.

indicative of a relatively large spread in the orientations of (111) planes with respect to the film normal, although sample Nos. 2 and 3 show a smaller spread as compared to that of sample No. 1. AFM measurements, however, reveal very similar morphological features for all the films with a mean roughness amplitude of $R_{a} \sim 50 \pm 10 \AA$ over a lateral width of $\sim 500 \AA$. This means that the magnetic ML layers are not atomically flat but have a significant waviness. In order to understand the origin of the surface waviness in the magnetic ML as well as the observed FWHM of the XRD rocking curves, cross-sectional TEM studies have been performed on one of the samples which will be described later in this article.

Magnetic hysteresis loops were measured at room temperature on the films by varying the applied magnetic field $\mathrm{H}$ in both parallel and perpendicular orientations with respect to the film plane. Figures 2 and 3 display such loops for sample Nos. 1 and 2. The shape of the hysteresis loops with $\mathrm{H}$ perpendicular to the film indicates that the easy axis of magnetization is along the film normal or at least close to it. However, the hysteresis loops measured with $\mathrm{H}$ parallel to the film plane are more complex to interpret. We speculate that (i) the magnetization of the film is canted with respect to film normal, and/or (ii) the film is magnetically inhomogeneous with some regions having their easy axis of magnetization in the film plane.

The various characteristic magnetization parameters determined from the hysteresis loops are listed in Table I. All three samples exhibit PMA with a saturation magnetization $\mu_{0} M_{S} \sim 0.9 \mathrm{~T}$ (averaged over the $\mathrm{Co} / \mathrm{Ni}$ multilayer volume). From the area between the two magnetization curves, the effective anisotropy energy constant $K_{U}^{*}$ is calculated. Sample Nos. 2 and 3 exhibit larger $K_{U}^{*}$ values, perpendicular remanences $\left(M_{R^{\perp}} / M_{S}\right)$, and coercivities $\left(H_{C^{\perp}}\right)$ as compared to the corresponding values for sample No. 1 . These data are also consistent with the hysteresis loops observed in the parallel geometry which have smaller remanence $\left(M_{R \|} / M_{S}\right)$ and coercivity $\left(H_{C \|}\right)$ values for sample Nos. 2 and 3 than those of sample No. 1. Further the value of $K_{U}^{*} d$ (the product 
TABLE I. XRD parameters and magnetic characteristics of $[\mathrm{Co}(2 \AA) / \mathrm{Ni}(7 \AA)]_{20}$ multilayer samples.

\begin{tabular}{|c|c|c|c|c|c|c|c|}
\hline Sample & $\begin{array}{c}\mathrm{FWHM} \\
\mathrm{Au} / \mathrm{Ag}\end{array}$ & $\begin{array}{c}\text { FWHM } \\
\mathrm{Co} / \mathrm{Ni}\end{array}$ & $\begin{array}{c}K_{U}^{*} \\
\left(\mathrm{MJ} / \mathrm{m}^{3}\right)\end{array}$ & $\begin{array}{c}H_{C \perp} \\
(\mathrm{kA} / \mathrm{m})\end{array}$ & $\begin{array}{c}H_{C \|} \\
(\mathrm{kA} / \mathrm{m})\end{array}$ & $\begin{array}{c}M_{R \perp} / M_{S} \\
(\%)\end{array}$ & $\begin{array}{c}M_{R \|} / M_{S} \\
(\%)\end{array}$ \\
\hline $\begin{array}{l}\text { No. } 1 \\
\text { HF-etched } \mathrm{Si}(111) \\
100 \AA \mathrm{Au} / 100 \AA \mathrm{Ag} \\
\text { buffer layers }\end{array}$ & $1.9^{\circ}$ & $1.8^{\circ}$ & 0.18 & 39 & 20 & 73 & 21 \\
\hline $\begin{array}{l}\text { No. } 2 \\
\mathrm{NH}_{4} \mathrm{~F} \text {-etched } \mathrm{Si}(111) \\
100 \AA \mathrm{Au} / 100 \AA \mathrm{Ag} \\
\text { buffer layers }\end{array}$ & $1.6^{\circ}$ & $1.5^{\circ}$ & 0.26 & 68 & 20 & 82 & 16 \\
\hline $\begin{array}{l}\text { No. } 3 \\
\mathrm{NH}_{4} \mathrm{~F} \text {-etched } \mathrm{Si}(111) \\
500 \AA \mathrm{Au} / 500 \AA \mathrm{Ag} \\
\text { buffer layers }\end{array}$ & $1.4^{\circ}$ & $1.4^{\circ}$ & 0.27 & 85 & 16 & 82 & 15 \\
\hline
\end{tabular}

Accuracy of FWHM is $\pm 0.1^{\circ}$.

$K_{U}^{*}$-effective anisotropy energy constant.

$H_{C \|}, H_{C \perp}$-coercive fields in parallel and perpendicular field configurations, respectively.

$M_{R \|} / M_{S}, M_{R \perp} / M_{S}$-relative remanence magnetization in parallel and perpendicular configurations.

of the effective anisotropy constant and the bilayer period) is found to be $\sim 0.19 \mathrm{~mJ} / \mathrm{m}^{2}$ for sample Nos. 2 and 3 which is comparable to the value of $\sim 0.3 \pm 0.1 \mathrm{~mJ} / \mathrm{m}^{2}$ extrapolated from Fig. 1 of Ref. 1. These data, in conjunction with the $\mathrm{XRD} /$ rocking curve data, indicate that a smaller spread in the (111) planes of the Co/Ni ML results in a stronger PMA. Thus a further improvement in the orientation of the (111) planes of the Co/Ni ML should result in better PMA properties.

As mentioned earlier, AFM measurements on all three samples reveal very similar surface morphological features indicating that the magnetic ML layers are not atomically flat but have significant waviness. In order to understand the origin of these features, a cross-sectional TEM study on sample No. 3 has been undertaken. Figure 4 shows a low-resolution TEM micrograph of the sample viewed along the $\langle 110\rangle$ zone axis of $\mathrm{Si}$. The presence of waviness at both top and bottom surfaces of the Co/Ni ML film is obvious with the waviness characterized by cusplike valley regions and relatively flatter

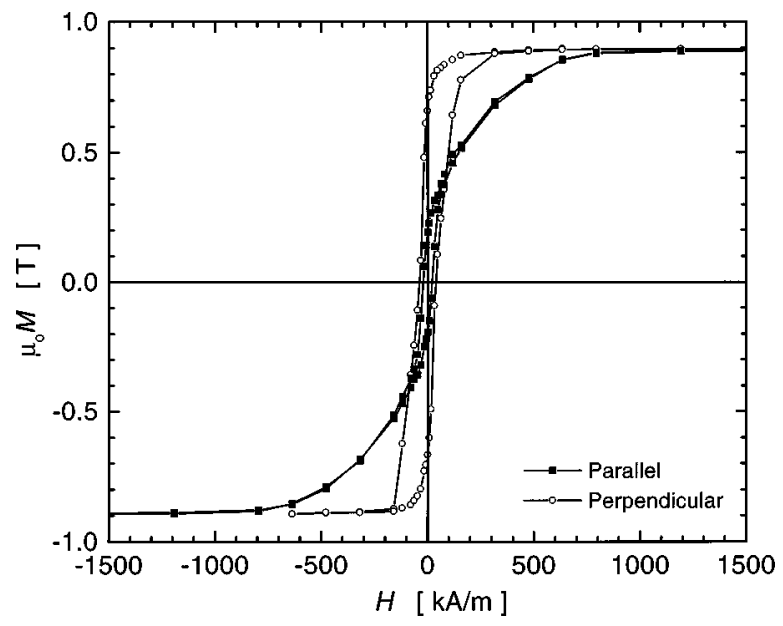

FIG. 2. Hysteresis loops for sample No. 1 with the applied magnetic field $\mathrm{H}$ oriented parallel and perpendicular to the film plane. hill regions. The average surface roughness amplitude $(\sim 40$ $\AA$ ) from the micrograph agrees with the AFM determination. These modulations of the magnetic layers are apparently a continuation of the surface waviness from the underneath $\mathrm{Au} / \mathrm{Ag}$ buffer layer which arises from the three-dimensional growth of $\mathrm{Ag}$ on hydrogen-terminated $\mathrm{Si}(111)$ at room temperature. $^{7}$ However, AFM measurements (not shown here) on a sample consisting of only $\mathrm{Au}(500 \AA) / \mathrm{Ag}(500 \AA)$ layers without any magnetic ML exhibited a smaller amplitude of $R_{a} \sim 35 \AA$ over a lateral width of 500-600 $⿱$. Thus the deposition of the magnetic ML seems to further enhance the waviness of the ML/Au interface. Perhaps this can be understood in terms of the difference in surface free energy $(\gamma)$ between $\mathrm{Au}\left(\sim 1200 \mathrm{erg} / \mathrm{cm}^{2}\right)$ and the magnetic elements $\mathrm{Co}$, Ni $\left(\sim 1800 \mathrm{erg} / \mathrm{cm}^{2}\right){ }^{8}$ Since $\gamma$ of the magnetic elements is much greater than $\gamma$ for Au the tendency will be for the magnetic layers not to wet with $\mathrm{Au}$, but for $\mathrm{Au}$ to creep into the magnetic layers and cause an increase in the amplitude of the interfacial waviness.

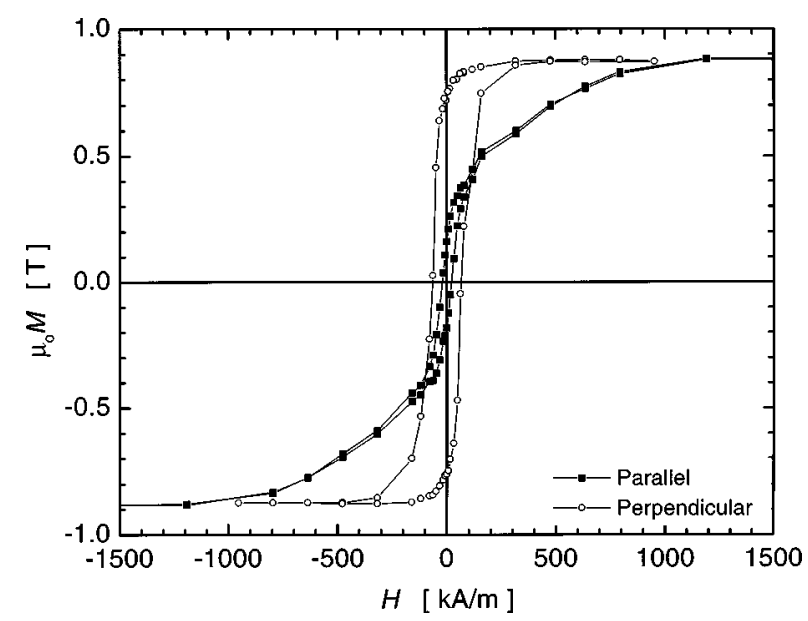

FIG. 3. Hysteresis loops for sample No. 2 with the applied magnetic field $\mathrm{H}$ oriented parallel and perpendicular to the film plane. 


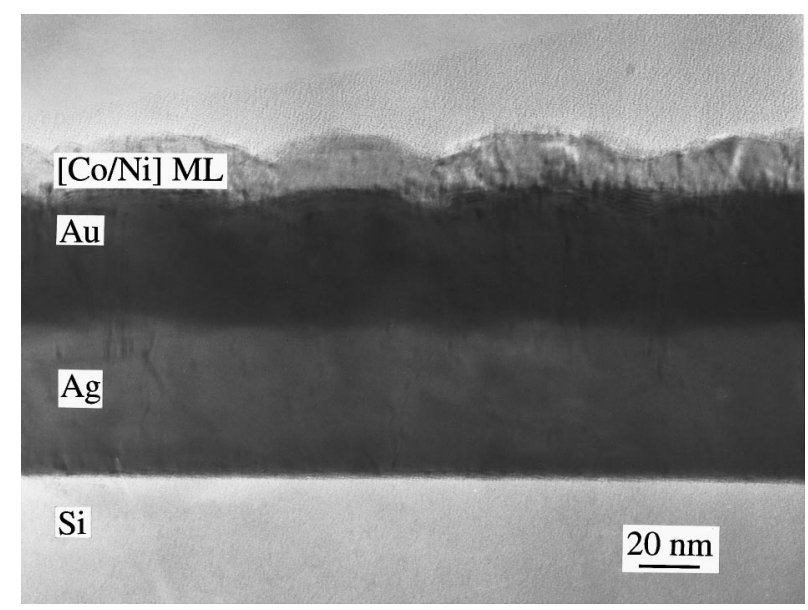

FIG. 4. Low-resolution cross-sectional TEM image viewed along $\operatorname{Si}\langle 110\rangle$ azimuth of sample No. 3.

A model developed by Bruno 9 can be utilized to discuss how the surface/interfacial roughness modifies the magnetic anisotropies of our $\mathrm{Co} / \mathrm{Ni} \mathrm{ML}$ films. According to their model a rough film is characterized by two parameters: one is the roughness $\sigma$, which is the mean square deviation from an ideally flat surface, and the other is the correlation length $\zeta$, i.e., the average lateral size of the flat areas on the surface. They have shown that the change in demagnetizing field is given by $\Delta H_{d} / H_{d}=-(3 / 2 t) \sigma[1-f(2 \pi \sigma / \zeta)]$, where $H_{d}$ is the demagnetizing field due to shape anisotropy, $t$ is the film thickness, and $f(2 \pi \sigma / \zeta)$ is a function that varies between 0 and 1. Similarly, any other surface/interface induced uniaxial anisotropy field $H_{K_{U}}$ is modified according to the equation $\Delta H_{K_{U}} / H_{K_{U}}=-2 \sigma / \zeta$. Using the measured values of $\sigma$ and $\zeta$ of $\sim 40$ and $\sim 500 \AA$ for sample No. $3, \Delta H_{d} / H_{d}=-0.1$ and $\Delta H_{K_{U}} / H_{K_{U}}=-0.16$. Although a reduction of $H_{K_{U}}$ is not favorable for enhancing the PMA, the reduction in $H_{d}$ favors an out-of-plane alignment of the magnetization.

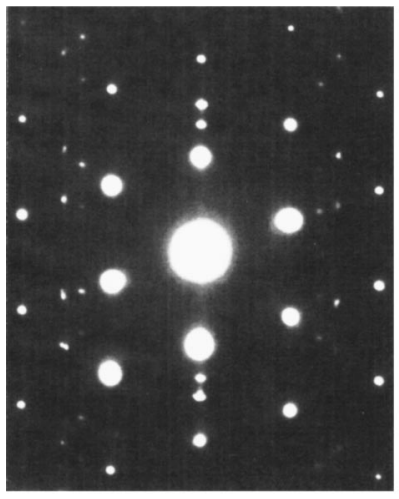

(a)

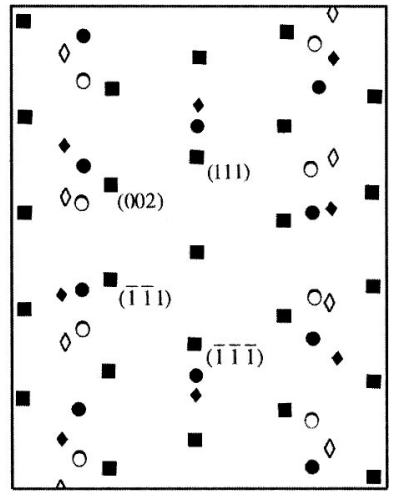

(b)
FIG. 5. (a) Selected area diffraction pattern (SADP) of overlapping Si, $\mathrm{Au} / \mathrm{Ag}$ buffer layer and $\mathrm{Co} / \mathrm{Ni} \mathrm{ML}$ regions. (b) Indexed pattern of (a) with $\square, \boldsymbol{b}, \boldsymbol{b}$ being primary spots of $\mathrm{Si}, \mathrm{Au} / \mathrm{Ag}$, and $\mathrm{Co} / \mathrm{Ni}$, respectively. $\bigcirc$ and $\diamond$ are from $180^{\circ}$ twins of $\mathrm{Au} / \mathrm{Ag}$ and $\mathrm{Co} / \mathrm{Ni}$, respectively.

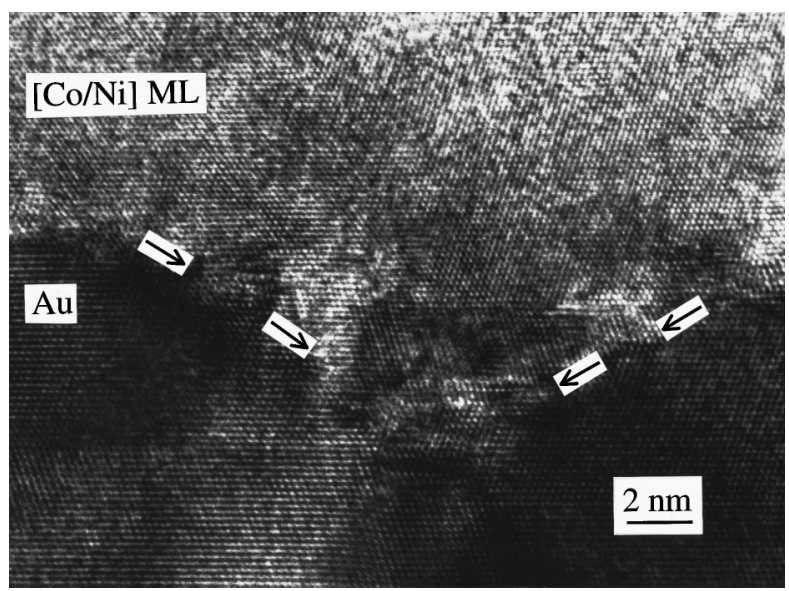

FIG. 6. High-resolution TEM image of $\mathrm{Co} / \mathrm{Ni}$ multilayer/Au interface near a cusplike valley region in Fig. 4. The arrows indicate the interface.

Figure 5 shows the selected area diffraction pattern (SADP) and the corresponding indexed pattern from an interfacial region of the $\mathrm{Si}$ substrate, the $\mathrm{Au} / \mathrm{Ag}$ buffer layer, and the $\mathrm{Co} / \mathrm{Ni}$ multilayers along the $\langle 1 \overline{1} 0\rangle$ zone axis of $\mathrm{Si}$. The epitaxial orientations are $\mathrm{Si}(111)\|\mathrm{Au} / \mathrm{Ag}(111)\|$ $\mathrm{Co} / \mathrm{Ni}(111)$ and $[1 \overline{1} 0]_{\mathrm{Si}}\left\|[1 \overline{1} 0]_{\mathrm{Au} / \mathrm{Ag}}\right\|[1 \overline{1} 0]_{\mathrm{Co} / \mathrm{Ni}}$. One also observes diffraction spots corresponding to $180^{\circ}$ twins of $\mathrm{Au} / \mathrm{Ag}$ and $\mathrm{Co} / \mathrm{Ni}$ with orientation relations of $\mathrm{Si}(111)\|\mathrm{Au} / \mathrm{Ag}(111)\| \mathrm{Co} / \mathrm{Ni}(111)$ and $[1 \overline{1} 0]_{\mathrm{Si}}\left\|[\overline{1} 10]_{\mathrm{Au} / \mathrm{Ag}}\right\|$ $[\overline{1} 10]_{\mathrm{Co} / \mathrm{Ni}}$ in agreement with the RHEED observations. The epitaxial growth between $\mathrm{Ag}$ and $\mathrm{Si}$ can be explained by the 4:3 coincident growth ${ }^{6}$ while the epitaxy between $\mathrm{Au}$ and $\mathrm{Ag}$ results from a very close lattice match between $\mathrm{Au}$ and $\mathrm{Ag}$ (lattice misfit $\sim 0.2 \%$ ). On the other hand there is a large lattice mismatch of $\sim 15 \%$ at the interface between $\mathrm{Au}$ and either Co or Ni. Thus one can expect that the lattice misfit will be accommodated by the formation of misfit dislocations at the interface as well as in the magnetic layers. The presence of a large lattice mismatch between $\mathrm{Au}$ and $\mathrm{Co} / \mathrm{Ni}$ lattices can also lead to a three-dimensional growth of the magnetic layers on Au causing a further increase in the waviness of the magnetic film. ${ }^{10}$ Indeed, a high-resolution TEM image (see Fig. 6) of the interface near a cusplike valley region reveals several $180^{\circ}$ twins/twin boundaries in the $\mathrm{Au}$ buffer layer and numerous defects in the magnetic ML including dislocations, stacking faults, twins/boundaries, and other misaligned regions. We believe that these features account for the large FWHM measured for the XRD rocking curves, and also for the presence of possible magnetic inhomogeneities in the $\mathrm{Co} / \mathrm{Ni} \mathrm{ML}$ in all three samples. As previously speculated, decreasing the waviness of the ML films should result in better orientation of the (111) planes of the multilayers and correspondingly improve its PMA properties. This can be achieved by starting with a flatter buffer layer. In a previous study ${ }^{7} 500-\AA$-thick $\mathrm{Ag}$ films when annealed at $275^{\circ} \mathrm{C}$ for $1 \mathrm{~h}$ under ultrahigh vacuum (UHV) conditions showed a smoother morphology $\left(R_{a}<10 \AA\right)$ with a significantly reduced FWHM of the (111) XRD peak. Also using $\mathrm{Cu}$ as a buffer layer instead of Au could possibly mini- 
mize the waviness in the multilayers since $\mathrm{Cu}$ 's surface free energy $\left(\gamma \approx 1400 \mathrm{erg} / \mathrm{cm}^{2}\right.$ ) is closer to that of $\mathrm{Ni}$ and $\mathrm{Co}$ and the lattice mismatch between $\mathrm{Cu}$ and the magnetic element is reduced $(\sim 2.1 \%$ with $\mathrm{Co}$ and $\sim 2.8 \%$ with Ni). Further work is in progress to fabricate $\mathrm{Co} / \mathrm{Ni} \mathrm{ML}$ with flatter interfaces and surfaces in order to assess the improvement in the PMA.

\section{CONCLUSIONS}

$[111]_{\text {fcc }}$ oriented $[\mathrm{Co}(2 \AA) / \mathrm{Ni}(7 \AA)]_{20}$ multilayers prepared by molecular beam epitaxy at room temperature on epitaxial $\mathrm{Au} / \mathrm{Ag}$ buffer layers grown on chemically etched $\mathrm{Si}(111)$ surfaces exhibit a perpendicular magnetic anisotropy. $\mathrm{NH}_{4} \mathrm{~F}$ etching of $\mathrm{Si}(111)$ leads to better oriented (111) planes of the $\mathrm{Au} / \mathrm{Ag}$ buffer layers and the $\mathrm{Co} / \mathrm{Ni}$ multilayers and stronger PMA characteristics as compared to a similar sample prepared on HF-etched $\mathrm{Si}(111)$. Cross-sectional TEM studies indicate that the magnetic multilayer film is not atomically flat but has a significant waviness at both top and bottom surfaces. The observed waviness originates in part from the waviness already present in the $\mathrm{Au}$ buffer layer surface and is further enhanced by the difference in surface free energies between $\mathrm{Au}$ and the magnetic elements. Fabricating flatter interfaces of $[111]_{\text {fcc }}$ oriented $[\mathrm{Co}(2 \AA) / \mathrm{Ni}(7 \AA)]_{20}$ ML should further improve the PMA properties.

\section{ACKNOWLEDGMENTS}

This work was supported by National Science Foundation Grant No. DMR-9400553. The transmission electron microscopy studies were carried out at the University of Michigan Electron Microbeam Analysis Laboratory.

${ }^{1}$ G. H. O. Daalderop, P. J. Kelly, and F. J. A. den Broeder, Phys. Rev. Lett. 68, 682 (1992).

${ }^{2}$ F. J. A. den Broeder, W. Hoving, and P. J. H. Bloemen, J. Magn. Magn. Mater. 93, 562 (1991).

${ }^{3}$ F. J. A. den Broeder, H. W. van Kesteren, W. Hoving, and W. B. Zeper, Appl. Phys. Lett. 61, 1468 (1992).

${ }^{4}$ P. Dumas, Y. J. Chabal, and G. S. Higashi, Phys. Rev. Lett. 65, 1124 (1990).

${ }^{5}$ G. S. Higashi, R. S. Becker, Y. J. Chabal, and A. J. Becker, Appl. Phys. Lett. 58, 1656 (1991).

${ }^{6}$ R. Naik, C. Kota, B. U. M. Rao, and G. W. Auner, J. Vac. Sci. Technol. A 12, 1832 (1994).

${ }^{7}$ R. Naik, G. W. Auner, S. Gebremariam, A. Tatham, B. U. M. Rao, Y. S. Lu, Z. L. Wu, and P. K. Kuo, Mater. Res. Soc. Symp. Proc. 355, 613 (1995).

${ }^{8}$ A. Zangwill, Physics at Surface (Cambridge University Press, Cambridge, 1989), p. 11.

${ }^{9}$ P. Bruno, J. Appl. Phys. 64, 3153 (1988); C. Chappert and P. Bruno, J. Appl. Phys. 64, 5736 (1988).

${ }^{10}$ A. G. Cullis, Mater. Res. Bull. 21, 21 (1996). 